\title{
Acquisition and propagation of spatial constraints based on qualitative information
}

\author{
AUTHOR(S):
}

Sogo, T; Ishiguro, H; Ishida, T

\section{CITATION:}

Sogo, T ...[et al]. Acquisition and propagation of spatial constraints based on qualitative information. IEEE TRANSACTIONS ON PATTERN ANALYSIS AND MACHINE INTELLIGENCE 2001, 23(3): 268-278

\section{ISSUE DATE:}

2001-03

URL:

http://hdl.handle.net/2433/50314

\section{RIGHT:}

(c) 2001 IEEE. Personal use of this material is permitted. However, permission to reprint/republish this material for advertising or promotional purposes or for creating new collective works for resale or redistribution to servers or lists, or to reuse any copyrighted component of this work in other works must be obtained from the IEEE. 


\title{
Acquisition and Propagation of Spatial Constraints Based on Qualitative Information
}

\author{
Takushi Sogo, Student Member, IEEE, Hiroshi Ishiguro, Member, IEEE, and \\ Toru Ishida, Senior Member, IEEE
}

\begin{abstract}
In robot navigation, one of the important and fundamental issues is to find positions of landmarks or vision sensors located around the robot. This paper proposes a method for reconstructing qualitative positions of multiple vision sensors from qualitative information observed by the vision sensors, i.e., motion directions of moving objects. In order to directly acquire the qualitative positions of points, the method proposed in this paper iterates the following steps: 1) observing motion directions (left or right) of moving objects with the vision sensors, 2) classifying the vision sensors into spatially classified pairs based on the motion directions, 3) acquiring three point constraints, and 4) propagating the constraints. Compared with the previous methods, which reconstruct the environment structure from quantitative measurements and acquire qualitative representations by abstracting it, this paper focuses on how to acquire qualitative positions of landmarks from low-level, simple, and reliable information (that is, "qualitative"). The method has been evaluated with simulations and also verified with observation errors.
\end{abstract}

Index Terms-Qualitative spatial representation, qualitative observation, spatially classified pair, three point constraint, constraint propagation, map building.

\section{INTRODUCTION}

$\mathrm{I}_{\mathrm{n}}^{\mathrm{N}}$ robotics and computer vision, acquisition of environment maps, which represent landmark positions and sensor positions, and their utilization are important research issues. Various quantitative and qualitative methods have been proposed so far. In general, quantitative methods [1], which use triangulation, stereo techniques, range sensors, etc., are based on the accumulation of precise metrical information. Triangulation, for example, is generally sensitive against sensory noise and accumulates errors, especially for some configurations of landmarks [2], so that proper error models and noise filtering techniques are necessary for quantitative methods [3], [4], [5], [6]. In contrast, it is expected that qualitative methods are not seriously affected by sensory noise and enable us to navigate robots in a wide environment, for example.

Levitt and Lawton reported a qualitative method for landmark-based robot navigation in an outdoor environment [7]. The robot observes the order of landmarks located around it and refers to a map to identify its qualitative location. The map indicates precise locations of the landmarks and defines qualitative locations, as shown in Fig. 1a. Besides this method, several works have been reported which utilize predefined qualitative maps and qualitatively utilize standard geometrical maps.

- T. Sogo and T. Ishida are with the Department of Social Informatics, Kyoto University, Sakyo-ku, Kyoto 606-8501, Japan.

E-mail: sogo@kuis.kyoto-u.ac.jp, ishida@i.kyoto-u.ac.jp.

- H. Ishiguro is with the Department of Computer and Communication Sciences, Wakayama University, 930 Sakaedani, Wakayama 640-8510, Japan. E-mail: ishiguro@sys.wakayama-u.ac.jp.

Manuscript received 21 July 2000; revised 30 Oct. 2000; accepted 12 Nov. 2000.

Recommended for acceptance by H. Christensen.

For information on obtaining reprints of this article, please send e-mail to: tpami@computer.org, and reference IEEECS Log Number 110276.
On the other hand, acquisition of the qualitative map itself is also necessary. Most of the previous works acquire qualitative landmark positions from observed quantitative information in the same way as triangulation, for example. However, the acquired map may not be consistent especially in a large-scale environment since the sensory data is noisy. A method which acquires qualitative landmark positions from more low-level and reliable information would be useful for robot navigation, etc., however, such a method has not been proposed so far.

Several methods have been proposed which acquire qualitative spatial representation by quantitative observation. Yeap developed a method for acquiring a cognitive map based on $2 \frac{1}{2}$-D representation of local areas [8]. The map is acquired with range sensors. Kuipers and Byun proposed a method for acquiring qualitative representation by exploration of a robot [9]. The representation consists of corridors and intersections recognized from sensory input. These methods deal with abstraction problems from perceptual information of a real world into qualitative representation and discuss how to integrate local representations into a global representation.

In this paper, we propose a method for reconstructing qualitative positions of landmarks from qualitative information acquired by visual observation [10]. The method observes motion directions of moving objects in an environment from several landmarks, as shown in Fig. 2a. While the objects move around the environment, the method acquires qualitative positions of the landmarks with several rules based on geometrical constraints. Generally, we consider that qualitative information is abstracted from quantitative information. However, the correctness of the qualitative information obviously depends on measurement methods. We use motion directions of moving objects as qualitative information since they are stably obtained by 


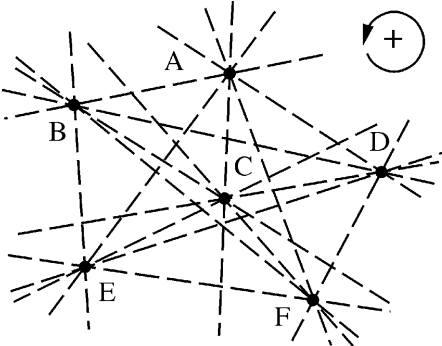

(a)

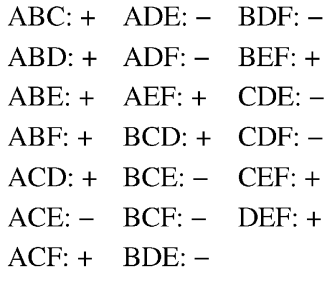

(b)
Fig. 1. Qualitative representation of positions. (a) An example configuration of points. (b) The corresponding qualitative spatial model.

tracking the objects for a sufficiently long time. Thus, compared with the previous acquisition methods, this paper focuses on how to acquire qualitative positions of landmarks from low-level, simple, and reliable information.

As an application of the method proposed in this paper, we consider a distributed vision system (DVS) [11], [12]. The DVS consists of multiple vision sensors embedded in an environment, as shown in Fig. 3, called vision agents, and successfully navigates robots based on visual information. Although the DVS does not use a geometrical map in robot navigation, qualitative positions of the sensors may be useful for coarse path planning of the robots. The proposed method can be applied to acquisition of the qualitative sensor positions in the DVS.

However, it is not only for the DVS. It can acquire qualitative maps of landmarks in general robot navigation. Suppose the robot has an omnidirectional vision sensor [13], observes motion directions of landmarks in the omnidirectional retina, as shown in Fig. 2b. If the robot can identify all of the landmarks or keep track of them anywhere in the environment, it can acquire the qualitative landmark positions with this method by observing their motion directions. In addition, the acquired map can be used for map-based robot navigation [7]. Thus, the method solves one of the general and fundamental problems in robot navigation and map building. Furthermore, the method is an algorithm for localizing multiple points based on analysis of geometrical constraints and can be applied to other domains as well as robotics. For example, it may be

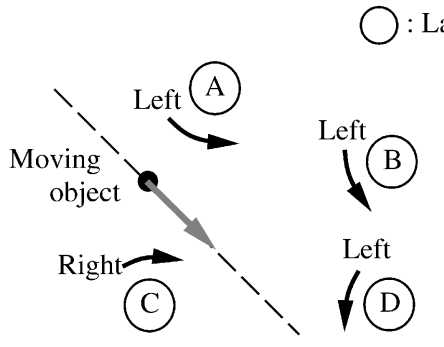

(a)

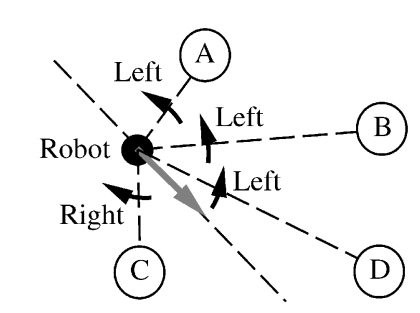

(b)
Fig. 2. Observation for acquiring qualitative positions. (a) A sensor at each landmark observes motion directions of moving objects in the environment. (b) A moving robot observes motion directions of landmarks.

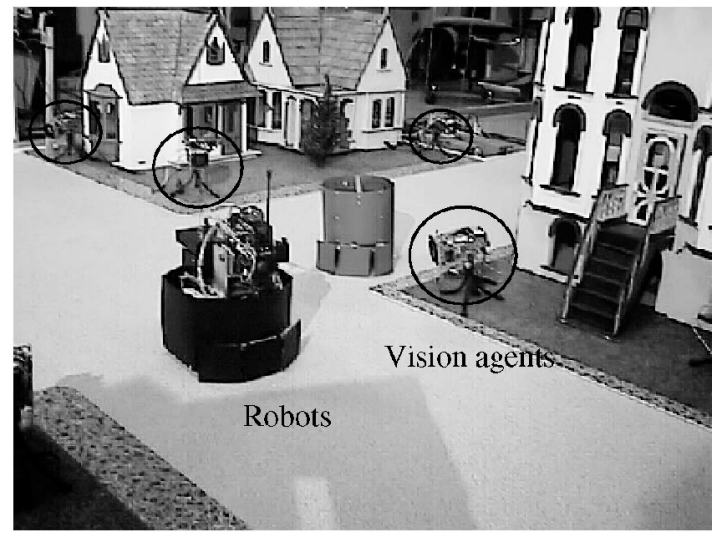

Fig. 3. Distributed vision system. The vision agents embedded in the environment navigate mobile robots.

used for coarse localization of sensors in surveillance applications using multiple cameras, radars, and so on.

In the following sections, we first introduce qualitative representation and qualitative observation in Section 2, then provide the detailed process for acquiring qualitative positions of landmarks in Section 3. Section 4 discusses the sensitivity of quantitative methods and the advantages of our method. Finally, we evaluate the proposed method with simulations in Section 5.

\section{Qualitative Representation AND Qualitative Observation}

\subsection{Qualitative Spatial Model}

In our method, the positions of points (in the remaining sections, we refer to landmarks as "points") are represented with relative positions with respect to lines passing over arbitrary two points, as shown in Fig. 1a. This is one of the simplest representation and various methods have been proposed in the field of qualitative spatial reasoning [14], [15], [16], [17]. This representation can be used for mapbased robot navigation [7].

Fig. 1b, called a qualitative spatial model, is a formal representation of the qualitative positions of the points shown in Fig. 1a. The model consists of several components, each of which represents positional relations among arbitrary three points as follows [18] (see Fig. 4):

- $\quad p_{i} p_{j} p_{k}=+$ if $p_{i} \rightarrow p_{j} \rightarrow p_{k}$ lie in counterclockwise,

- $\quad p_{i} p_{j} p_{k}=-$ if $p_{i} \rightarrow p_{j} \rightarrow p_{k}$ lie in clockwise,

where $p_{i}, p_{j}$, and $p_{k}$ are arbitrary three points. In the case of six points, as shown in Fig. 1 , these $\left(\begin{array}{l}6 \\ 3\end{array}\right)=20$ components are

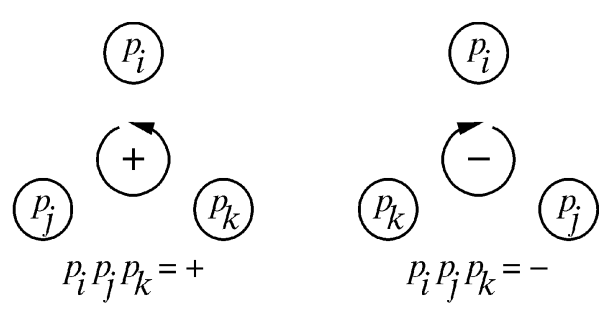

Fig. 4. Qualitative representation of positional relations among three points. 


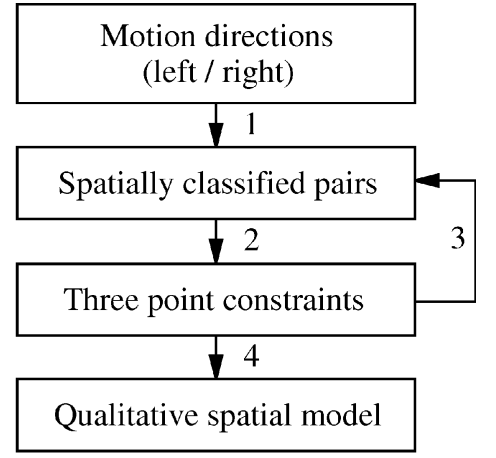

Fig. 5. Process for acquiring the qualitative spatial model.

needed to represent all positional relations among the points.

The purpose of this paper is to propose a method to acquire the qualitative spatial model, as shown in Fig. 1b by qualitative observation described in the following section.

\subsection{Qualitative Observation}

The qualitative spatial model is acquired by observing motion directions of moving objects from each point. In the case of Fig. 2a, for example, vision sensors at the points $A$ through $D$ simultaneously observe instant motion directions of the object. When the projection of the moving object moves clockwise in the omnidirectional retina of a vision sensor, the motion is qualitatively represented as "right" and when it moves counterclockwise, it is represented as "left." Note that the same information can also be obtained by opposite observation, i.e., when a mobile robot observes motion directions of points, as shown in Fig. $2 b$.

With the observed motion directions, the points are classified into a spatially classified pair (SCP), which consists of a pair of point sets labeled "left" and "right." In the case of Fig. 2, an SCP " $\{A B D\},\{C\}$ " is acquired by observation, which means that there is a straight line that classifies the points into such a pair of point sets. By iterating the observation while the object moves around the environment, various SCPs are acquired except inconsistent ones. For example, an SCP " $\{A D\},\{B C\}$ " is inconsistent with the configuration of the points shown in Fig. 2, since there is no straight line which classifies the points into such a pair.

Note that if the sensor cannot determine the motion direction of the object, the sensor (point) will not appear in the $\mathrm{SCP}$. This means that the SCP has no information with respect to the point position. The qualitative spatial model is acquired from the SCPs, as described in the next section, however, the qualitative position of a point will not be acquired unless it observes the motion direction of the object.

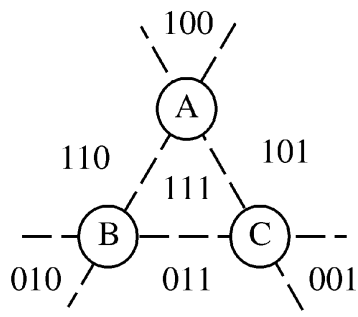

Fig. 6. Seven regions defined with three points.

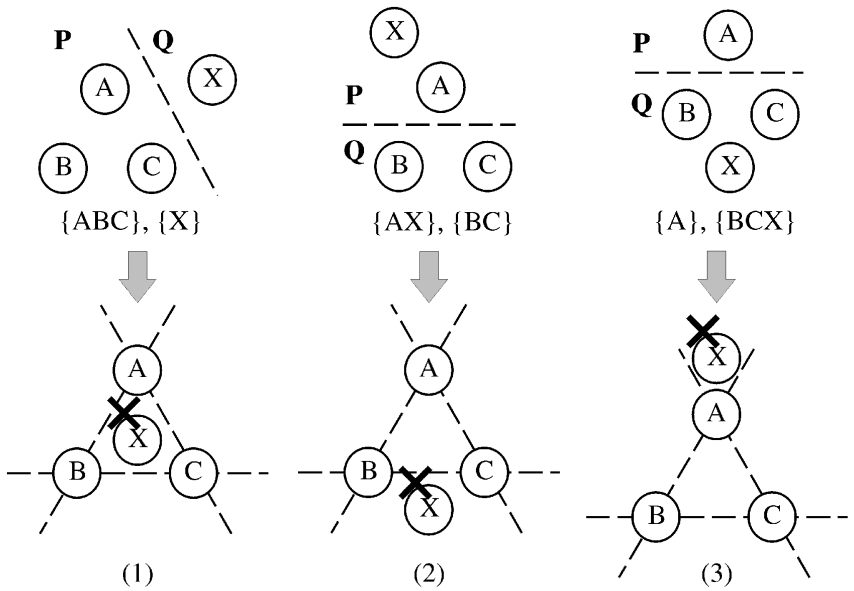

Fig. 7. Three point constraints. The crosses represent regions where $X$ is not located.

\section{Acquisition of the Qualitative Spatial MODEL}

\subsection{Overview}

SCPs represent geometrical constraints among positions of points, as described in the previous section. The qualitative spatial model, an example of which is shown in Fig. 1b, is acquired from the SCPs by iterating the following steps (see Fig. 5):

1. Acquire an SCP by qualitative observation (see Section 2.2).

2. Acquire three point constraints (3PCs) from the SCP.

3. Classify the points into new SCPs based on the 3PCs, and acquire new 3PCs (constraint propagation).

4. Transform the 3PCs into the qualitative spatial model.

The following sections explain Steps 2, 3, and 4 .

\subsection{Acquisition of Three Point Constraints}

In order to determine the qualitative positions of the points, our method checks possible positions of the fourth point with respect to a triangle consisting of three points. Since a triangle is the minimum component to represent closed regions, we can represent the qualitative positions of all points by combining the triangles.

Let us consider four points $A, B, C$, and $X$. The qualitative position of $X$ with respect to $A, B$, and $C$ is represented with one of the seven regions defined with three lines $A B, A C$, and $B C$, and encoded, as shown in Fig. 6. Several constraints which limit possible regions of $X$ are acquired from SCPs based on geometrical constraints. Suppose $A, B, C$, and $X$ are classified into SCPs " $\mathbf{P}, \mathbf{Q}$ " in various ways, as shown in Fig. 7. Considering positional symmetry of the points, the geometrical constraints are summarized into the following cases:

1. When $\mathbf{P}$ includes $A, B$, and $C$ :

If $\mathbf{P}$ also includes $X$, there is no constraint on the position of $X$. If $\mathbf{Q}$ includes $X$, which is the other set to $\mathbf{P}, X$ is not located in the region 111 (see Fig. 7 (1)). 


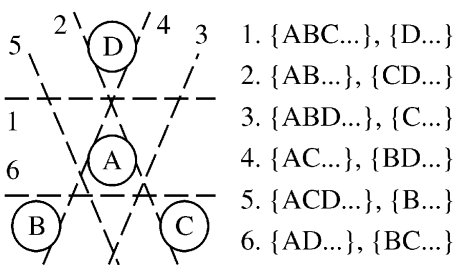

(a)

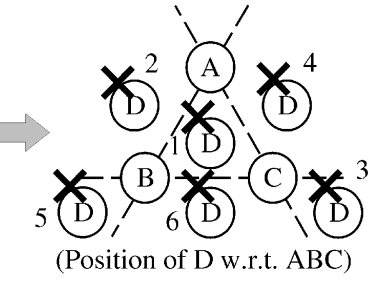

(b)
Fig. 8. An example of possible (a) SCPs and (b) 3PCs. For general configuration of four points, six SCPs are acquired by observation and transformed into six 3PCs.

2. When $\mathbf{P}$ includes $A$ and $\mathbf{Q}$ includes $B$ and $C$ :

If $\mathbf{P}$ also includes $X$, it is not located in the region 011 (see Fig. 7 (2)). If $\mathbf{Q}$ includes $X$, it is not located in the region 100 (see Fig. 7 (3)).

We call these constraints three point constraints (3PCs). In general, there are six different SCPs with respect to arbitrary four points, as shown in Fig. 8a. The six SCPs are acquired by observation if motion directions of objects are sufficiently observed from the points, and they are transformed into six 3PCs with respect to each point's position, as shown in Fig. 8b, which uniquely determine the region of the point. In the same way, the qualitative positions of all points are determined when all possible SCPs are acquired by observation.

\subsection{Constraint Propagation}

Various SCPs and 3PCs are acquired by iterating observation of motion directions of objects, however, in practice there are some limitations of observation. For example, vision sensors cannot observe objects in distant locations and behind walls. In this case, the observation will not provide sufficient SCPs (and 3PCs) for reconstructing a complete qualitative spatial model. However, the 3PCs acquired from SCPs provide further 3PCs. This can be considered as constraint propagation.

A simple example of the constraint propagation is as follows: Let us consider five points $A$ through $E$. When the positions of $D$ and $E$ have been uniquely determined with twelve 3PCs with respect to $A, B$, and $C$, as shown in Fig. 9a, the points $C, D$, and $E$, for example, are classified into the following SCP with the line $A B$ :

$$
\{D\},\{C E\} .
$$

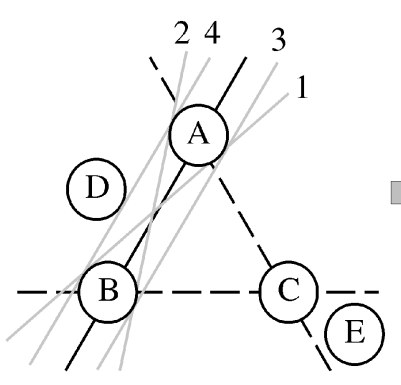

(a)

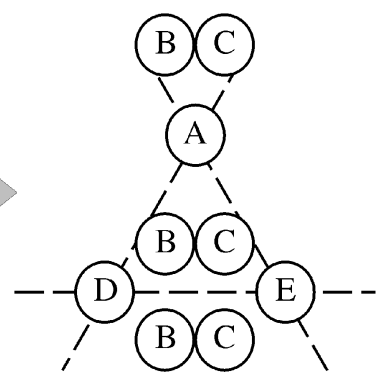

(b)
Fig. 9. An example of constraint propagation. (a) An example of positions. (b) Propagated positions.
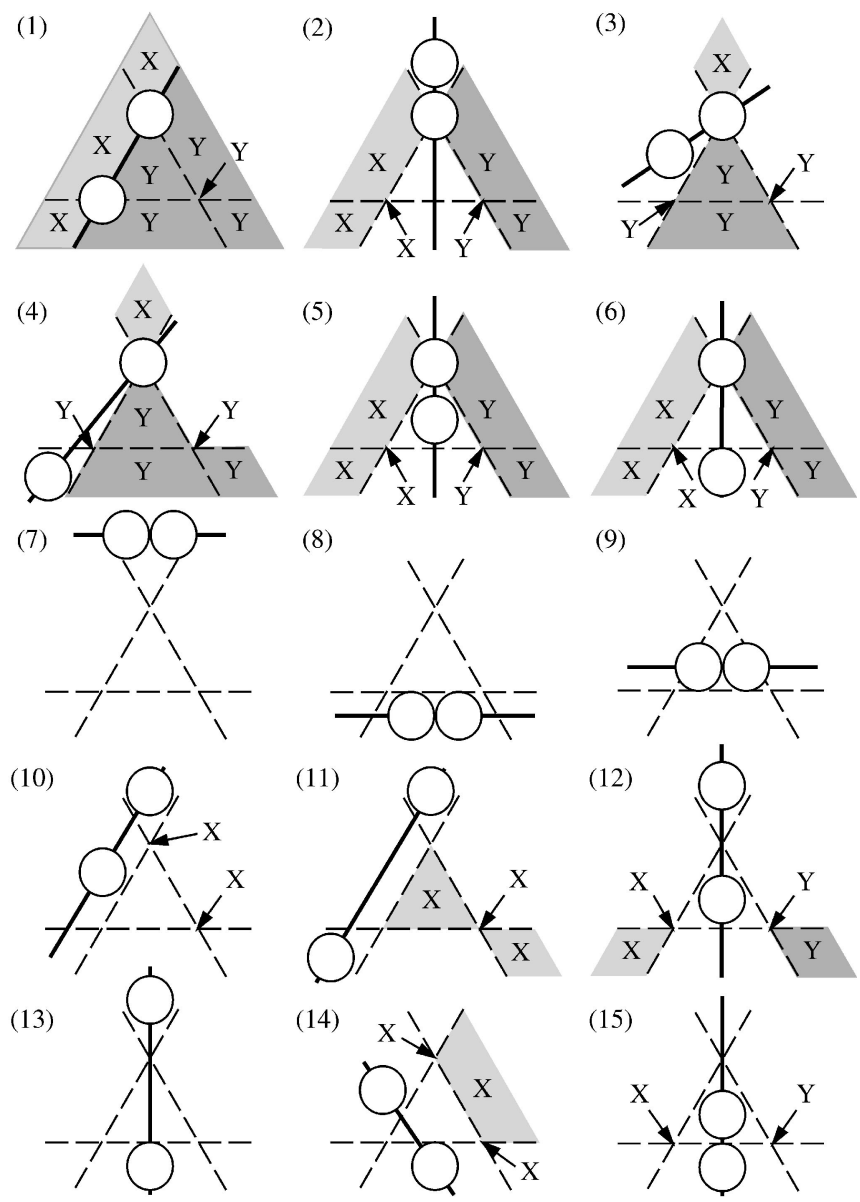

Fig. 10. Classifications for the constraint propagation.

Note that the notation of the qualitative positions in Figs. 9, 10, 11, and 12 is different from that of 3PCs, as shown in Fig. 7 and 8, for simple representation. That is, 3PCs originally represent regions where a point is not located, however, these figures indicate regions where a point is located.

Furthermore, there are four lines around the line $A B$ which classify the five points, including $A$ and $B$, into the following SCPs (the numbers correspond to those in Fig. 9a):

$$
\begin{aligned}
& \text { 1. }\{A D\},\{B C E\} \\
& \text { 2. }\{B D\},\{A C E\} \\
& \text { 3. }\{A B D\},\{C E\} \\
& \text { 4. }\{D\},\{A B C E\} .
\end{aligned}
$$

There are $\left(\begin{array}{l}5 \\ 2\end{array}\right)=10$ lines which pass over two points out of $A$ through $E$. Each line classifies the points into several

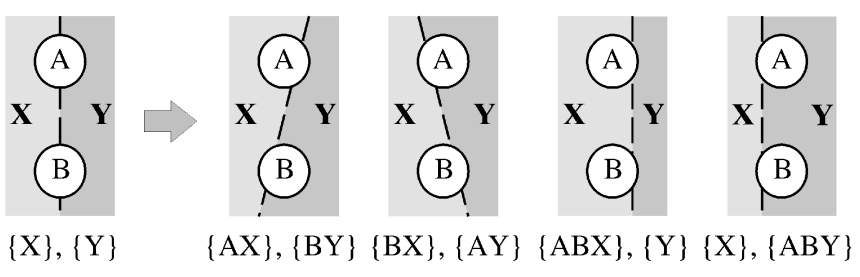

Fig. 11. SCPs including the points on the classifying line. 


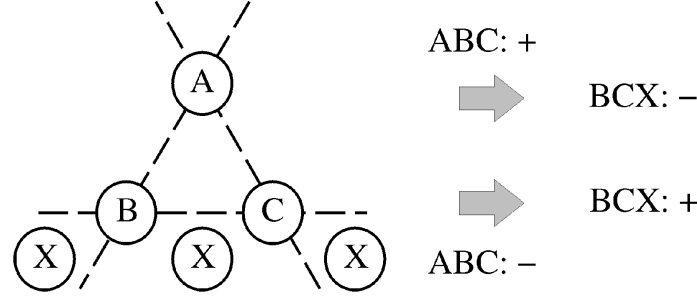

Fig. 12. Transformation into the qualitative spatial model.

SCPs in the same way. Consequently, the following seven different SCPs are acquired in the case of Fig. 9a:

$\begin{array}{ll}\{A B C D\},\{E\} & \{A C E\},\{B D\} \\ \{A B C E\},\{D\} & \{A D\},\{B C E\} \\ \{A B D\},\{C E\} & \{A E\},\{B C D\} \\ \{A C D\},\{B E\} . & \end{array}$

Then, these SCPs are transformed into several 3PCs, as described in Section 3.2. Fig. $9 \mathrm{~b}$ shows an example of possible positions of $B$ and $C$ with respect to $A, D$, and $E$ acquired from these SCPs.

\subsection{Formalization of the Constraint Propagation}

The process for acquiring new SCPs described in the previous section is as follows:

1. Acquire an SCP classified by a line passing over arbitrary two points (an example is the SCP (1) in the previous section).

2. Then, transform it into four SCPs including the two points (an example is the SCPs (2)).

This process can be formally summarized as follows:

Let us suppose that positions of several points (i.e., regions where they are located) with respect to a triangle $A B C$ have been uniquely determined with 3PCs. Then, a line is considered which passes over two of the points and classifies the other points into an SCP. Considering positional symmetry of the points, there are 15 kinds of selection of two points which the classifying line passes over, as shown in Fig. 10, where the circles indicate the selected two points and the points in the regions $\mathbf{X}$ and $\mathbf{Y}$ are classified into an SCP " $\{\mathbf{X}\},\{\mathbf{Y}\}$ " with the line. Fig. 10 (1) corresponds to the case in which the selected points are two of $A, B$, and $C$. Fig. 10 (2) through (6) correspond to the case in which one of the selected points are $A, B$, and $C$. Fig. 10 (7), (8), and (9) correspond to the case in which the selected points are located in the same region. Fig. 10 (10) through (15) correspond to the case in which the selected points are located in different regions. Note that no SCP is acquired in Fig. 10 (7), (8), (9), and (13), and an SCP " $\{\mathbf{X}\},\{\emptyset\}$ " is acquired in Fig. 10 (10), (11), and (14).

Then, SCPs, including the two points on the classifying line, are considered. Suppose the line $A B$ classifies the other points into an SCP " $\{\mathbf{X}\},\{\mathbf{Y}\}$." Although $A$ and $B$ are not included in the SCP in the above discussion, there are four lines which classify $A$ and $B$, as well as $\mathbf{X}$ and $\mathbf{Y}$ into the following SCPs (see Fig. 11):
$\{A \mathbf{X}\},\{B \mathbf{Y}\}$
$\{A B \mathbf{X}\}, \quad\{\mathbf{Y}\}$
$\{B \mathbf{X}\},\{A \mathbf{Y}\} \quad\{\mathbf{X}\}, \quad\{A B \mathbf{Y}\}$.

Thus, new SCPs can be acquired from 3PCs, then the SCPs are transformed into new 3PCs, as described in Section 3.2.

In the above discussion, the constraint propagation is performed when the positions of the points have been uniquely determined with 3PCs (i.e., each point is located in one of the seven regions of a triangle). However, even if they have not been uniquely determined, the constraint propagation can be performed with respect to the points each of which is located only in the region $\mathbf{X}$ or $\mathbf{Y}$ shown in Fig. 10. In the experimentation of Section 5, the constraint propagation is performed under such a situation.

\subsection{Transforming into the Qualitative Spatial Model}

The 3PCs are transformed into the qualitative spatial model (see Fig. 1b) as follows: For example, if the position of $X$ with respect to $A, B$, and $C$ has been determined with 3PCs, as shown in Fig. 12, then the order of $B C X(B \rightarrow C \rightarrow X)$ is determined to be opposite to that of $A B C(A \rightarrow B \rightarrow C)$; that is, $B C X=-$ if $A B C=+$, and $B C X=+$ if $A B C=-$. If the order of $A B C$ is given, the order of $B C X$ is uniquely determined. Consequently, all components of the qualitative spatial model are uniquely determined when six 3PCs with respect to each point's position have been acquired, as shown in Fig. 8b.

\subsection{Computational Costs}

In this section, we discuss the computational costs of the algorithm. Note that the discussion excludes the constraint propagation since its behavior is complicated.

As discussed in Section 3.2, qualitative positions of all points are determined when all possible SCPs are acquired by observation (see Fig. 8). The $k$-sets theory [19] gives the number of possible SCPs (i.e., the number of straight lines which classify the points into different SCPs) as follows: In the $k$-sets theory, it has been proved that the upper bound of the number of point sets, which contain at most $k$ points and are cut off by straight lines from $n$ points in a plane, is $k n(k<n / 2)$. The case of the SCPs is considered as the same problem for $k=n / 2$ (i.e., we consider all of the straight lines including those which classify the $n$ points into exact halves). It has not been proved for $k=n / 2$ so far, however, it is estimated to be $O\left(n^{2}\right)$ [20]. Thus, the number of possible SCPs is $O\left(n^{2}\right)$. In other words, our method has to observe different motion directions of objects $O\left(n^{2}\right)$ times to acquire the qualitative positions of $n$ points.

Next, the number of 3PCs which the method needs to check in order to acquire the qualitative spatial model is considered. If motion directions of objects are observed from all of $n$ points, every SCP obtained by the observation contains all of the $n$ points. Then, the algorithm chooses arbitrary three points out of $n$ points in the acquired SCP and checks 3PCs with respect to remaining $(n-3)$ points, as described in Section 3.2. Consequently, the algorithm checks $\left\{\left(\begin{array}{l}n \\ 3\end{array}\right)(n-3)\right\}=O\left(n^{4}\right)$ 3PCs for every SCP. Since the number of SCPs needed to acquire the positions of $n$ points 
is $O\left(n^{2}\right)$ as described above, the method checks $O\left(n^{6}\right) 3 \mathrm{PCs}$ to acquire the qualitative spatial model.

However, the number of 3PCs needed to represent the qualitative positions of points is far less than that. As described in Section 3.2, the qualitative position of a point with respect to arbitrary three points out of $(n-1)$ is uniquely determined with six 3PCs, as shown in Fig. 8. Therefore, $\left\{n \cdot\left(\begin{array}{c}n-1 \\ 3\end{array}\right) \cdot 6\right\}=O\left(n^{4}\right) 3 \mathrm{PCs}$ are needed to represent the qualitative positions of $n$ points. Since the number of 3PCs the method checks is $O\left(n^{6}\right)$ as described above, it redundantly checks many 3 PCs.

\subsection{Distributed Computation}

As described above, the computational costs of the proposed method are rather high. However, for practical implementation, we can expect to employ parallel computation using distributed computing resources. Let us suppose that each sensor has computational ability. The qualitative spatial model can be acquired in a distributed manner as follows:

1. Observe motion directions of objects simultaneously.

2. Exchange the motion directions with other sensors which observed the same object and acquire an SCP.

3. At each sensor, independently compute 3PCs and the components of the qualitative spatial model related to the sensor position.

In the distributed computation, it is expected that the method can acquire the qualitative spatial model even if the number of the sensors increases since, in practice, vision sensors do not observe objects in distant locations, and the computation of SCPs and 3PCs is performed only at the sensors which observed objects. However, the message exchange costs among the sensors may increase. In future work, problems in the distributed computation should be considered in more detail.

\section{Advantages of the Qualitative Method}

Theoretically, the qualitative spatial model can be also acquired by quantitative methods which transform metrical positions of points into qualitative positions. This section considers quantitative methods which directly acquire metrical positions of sensors from visual information without any other sensory information and discusses the advantages of the proposed method.

\subsection{Quantitative Method}

Let us consider the same scenario as the qualitative method, that is, multiple vision sensors measure their own metrical positions by observing azimuth angles without reference points of known positions, as shown in Fig. 13a. In the following discussion, we suppose that the positions of the sensors are directly measured from the azimuth angles.

The following equation represents the constraints among the sensor positions:

$$
\cos \angle_{i j k}=\frac{\left(\mathbf{p}_{i}-\mathbf{p}_{j}\right) \cdot\left(\mathbf{p}_{k}-\mathbf{p}_{j}\right)}{\left|\mathbf{p}_{i}-\mathbf{p}_{j}\right|\left|\mathbf{p}_{k}-\mathbf{p}_{j}\right|},
$$

where $\mathbf{p}_{i}, \mathbf{p}_{j}$, and $\mathbf{p}_{k}$ represent the positions of arbitrary three sensors on a $2 \mathrm{D}$ plane and $L_{i j k}$ is given by observing

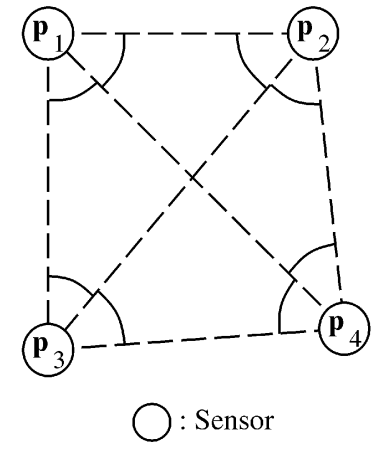

(a)

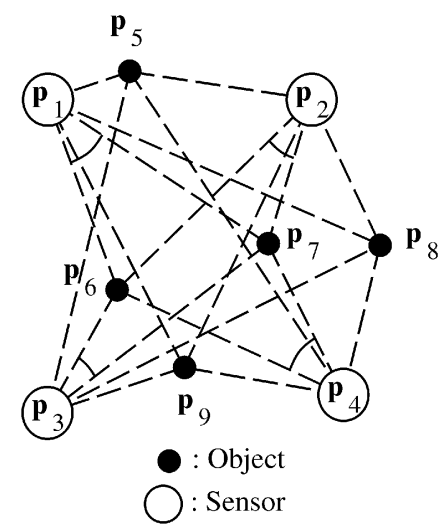

(b)
Fig. 13. Acquisition of quantitative sensor positions. (a) Each sensor observes other sensors. (b) Each sensor observes objects in the environment instead of sensors.

the angle between $\mathbf{p}_{i}$ and $\mathbf{p}_{k}$ from $\mathbf{p}_{j}$. If the sensors are very small and cannot observe each other, they observe objects in the environment instead, as shown in Fig. 13b. In this case, $\mathbf{p}_{j}$ represents the positions of the sensors, and $\mathbf{p}_{i}$ and $\mathbf{p}_{k}$ represent those of the objects.

Let us suppose that there are 10 sensors of unknown positions observing moving objects. In order to compute the metrical positions, the sensors have to measure azimuth angles to four objects, from which we obtain 30 equations based on (1). These nonlinear simultaneous equations are generally solved by numerical methods which compute approximate solutions using proper initial estimates [3]. This process is a kind of triangulation, which can be in general very sensitive against observation errors for some configurations of reference points (i.e., the objects) [2]. Therefore, proper error models are necessary for acquiring better solutions [4], [5]. Note that, in our scenario, the equations representing the sensor and object positions are more sensitive than those of conventional triangulation, since the positions of the reference points (objects) are also unknown. In addition, if the objects are not small points and have complex shapes, the measurements of azimuth angles to the objects may be noisy in practice. Therefore, iterative observation and noise filtering techniques are necessary [3], [6].

The qualitative spatial model can be obtained from the metrical positions of the sensors. However, the sensitive nonlinear equations and observation errors may yield wrong qualitative positions of the sensors in the model.

\subsection{Qualitative Method}

In the qualitative method, the acquisition process described in Section 3 itself is sensitive against errors of motion directions so that even a few errors may cause wrong qualitative positions in the qualitative spatial model. More specifically, observation errors may yield wrong SCPs and 3PCs as follows: If the observation error yields an inconsistent SCP (inconsistent classification, such as " $\{A D\},\{B C\}$ " in the case of Fig. 2), wrong 3PCs are acquired, which mistakenly determine the qualitative positions of points. In addition, only six kinds of 3PCs 

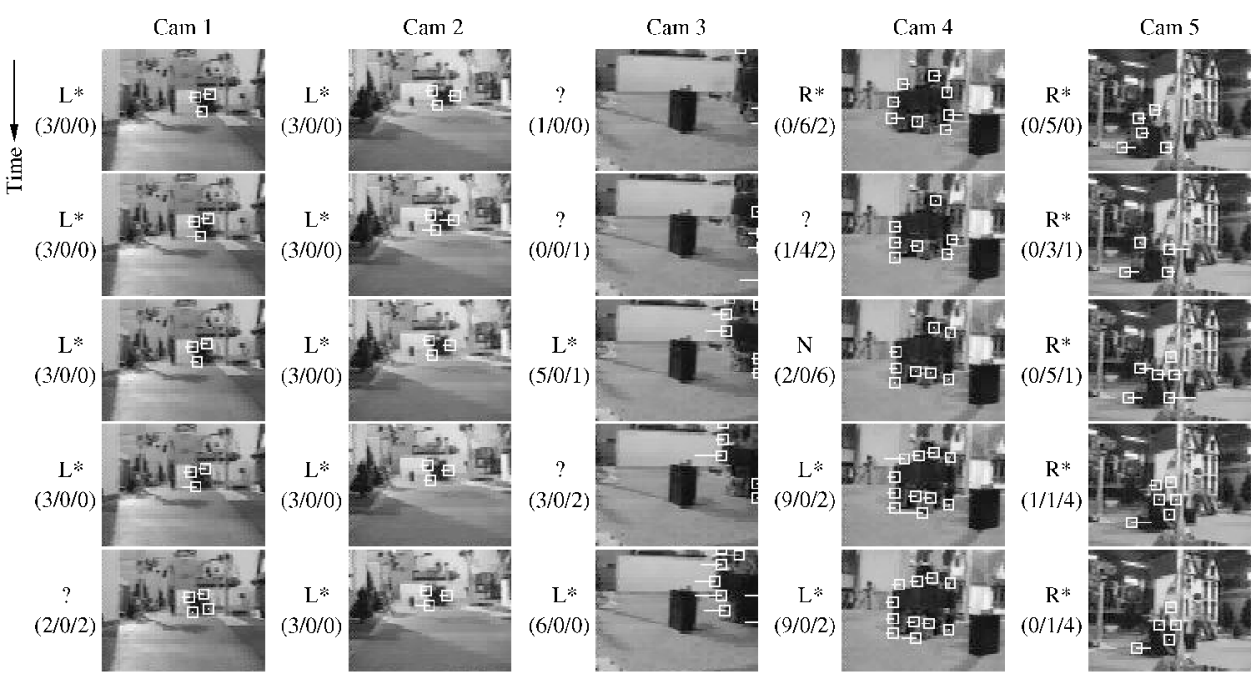

Fig. 14. Motion directions detected by background subtraction and template matching. Five successive images taken with each vision sensor are shown. The numbers in parentheses indicate $n_{L} / n_{R} / n_{N}$, and $L, R, N$, and "?" indicate the detected motion directions."*" indicates that the detected motion direction $(L$ or $R$ ) is correct.

should be acquired with respect to arbitrary four points, as shown in Fig. 8, however, inconsistent SCPs may give all of the seven 3PCs, from which correct qualitative positions cannot be acquired. For this problem, Section 5.3 provides a simple method which statistically eliminates wrong 3PCs.

Thus, the algorithm itself is sensitive against observation errors. However, observation of motion directions of moving objects is fairly stable in practice. Let us suppose the following method for detecting motion directions of a robot with multiple vision sensors:

1. Detect the robot by background subtraction.

2. Find several small regions, such as vertical edges which can be used for template matching (indicated with the white rectangles in Fig. 14).

3. Compute optical flows by template matching (the horizontal lines in the white rectangles indicate the optical flows).

4. Check the direction of each flow and determine the motion direction of the robot based on majority rule.

We have verified the above method with a model town made for the distributed vision system (see Fig. 3). There are five vision sensors, which observe a robot at a distance between $50 \mathrm{~cm}$ and $150 \mathrm{~cm}$ and take images of $160 \times 120$ pixels whenever the robot moves approximately $5 \mathrm{~cm}$. In this experimentation, we have taken 250 images in total and determined the motion direction $D \in\{L, R, N$, ? $\}$ (left, right, no motion, and unknown, respectively) based on the number of the templates as follows:

- $D=L$ if $n_{L} \geq 3$ and $n_{L} \geq 2\left(n_{R}+n_{N}\right)$,

- $\quad D=R$ if $n_{R} \geq 3$ and $n_{R} \geq 2\left(n_{L}+n_{N}\right)$,

- $D=N$ if $n_{N} \geq 3$ and $n_{N} \geq 2\left(n_{L}+n_{R}\right)$,

- otherwise $D=$ ?,

where $n_{L}$ and $n_{R}$ are the number of templates which moved left and right, respectively, and $n_{N}$ is that of no motion. A threshold for detecting the motion directions of the templates is 1 pixel.
Fig. 14 shows part of the experimental results, where five successive images taken with each vision sensor and estimated motion directions are shown. The above method correctly determined 113 motion directions ( $L^{*}$ and $R^{*}$ in Fig. 14) out of 250 and could not determine 137 motion directions (21 of which are no motion $(N)$ and 116 are unknown (?)). This means that the optical flow estimation by template matching provides correct motion directions if we refer to an enough number of templates. Furthermore, the intervals for taking images and the threshold for motion detection are fixed in this experimentation, however, if we dynamically adjust them according to the motion of the robot and use high-resolution vision sensors, the above method for detecting motion directions will provide better results. Note that the latter 137 results do not affect the correctness of the qualitative spatial model, as described in Section 2.2.

The method is the simplest one and there exist more sophisticated methods. We can expect that, in general, correct motion directions of objects are obtained if they are tracked for a sufficiently long time. Thus, it is possible to stably acquire qualitative motion directions by image processing and the qualitative method proposed in this paper is considered as an alternative to quantitative methods for acquiring qualitative positions of points by visual observation in a simple and stable manner.

\section{Experimental Results}

\subsection{Verification with a Simple Environment}

We have acquired the qualitative spatial model representing qualitative positions of vision sensors by the proposed method with simulations. First, we have verified the method with a simple environment. In the environment, there are 20 vision sensors and a moving object in a $20 \mathrm{~m} \times 20 \mathrm{~m}$ space, as shown in Fig. 15 The vision sensors have omnidirectional views and observe motion directions of the object in all directions whenever it randomly moves 


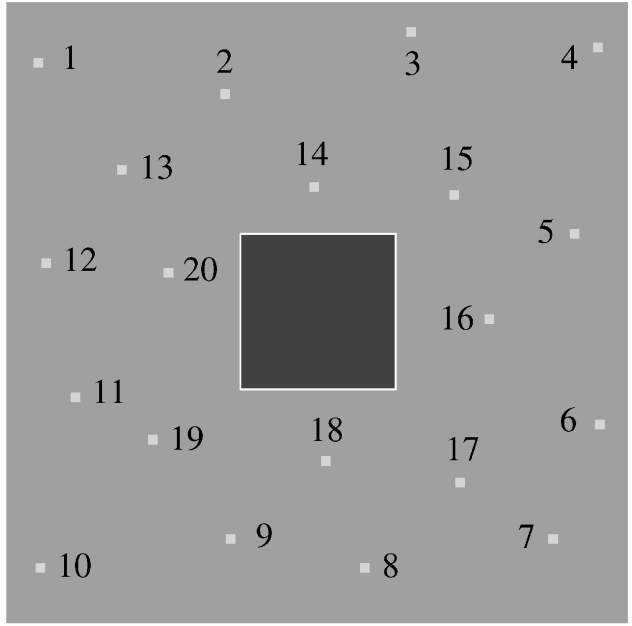

Fig. 15. Simple environment with 20 vision sensors and a moving object. The walls in the center of the environment obstruct the views of the sensors.

$1 \mathrm{~m}$ on the light gray region in Fig. 15. However, they cannot observe the object at a distance of more than $10 \mathrm{~m}$ or behind walls (indicated with the white lines in Fig. 15). In this experiment, the number of components of the qualitative spatial model (represented with " + " and "-," as shown in Fig. $1 b)$ is $\left(\begin{array}{c}20 \\ 3\end{array}\right)=1,140$. However, the proposed method cannot acquire all of the components since the sensors cannot observe sufficient motion directions of the object. It is estimated from the configuration of the sensors that about 560 components will be acquired without constraint propagation.

Fig. 16 shows the average number of acquired components over five runs. With 5,000 observations, the method has determined the directions ("+" or "-") of 490 components without constraint propagation. On the other hand, it has determined the directions of 969 components with constraint propagation, which are twice as many as those acquired without constraint propagation, and 85 percent of all components.

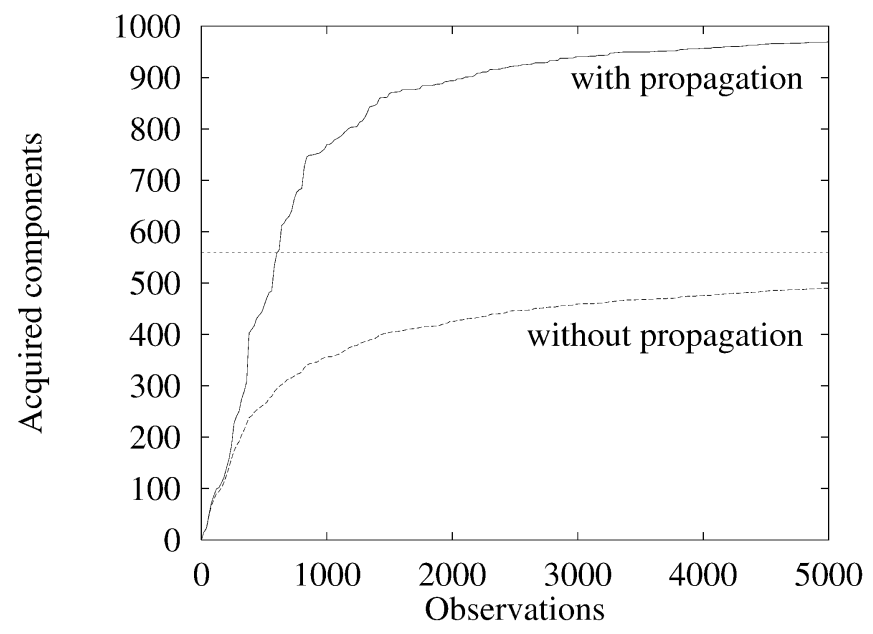

Fig. 16. The number of components acquired with 20 vision sensors in the simple environment.

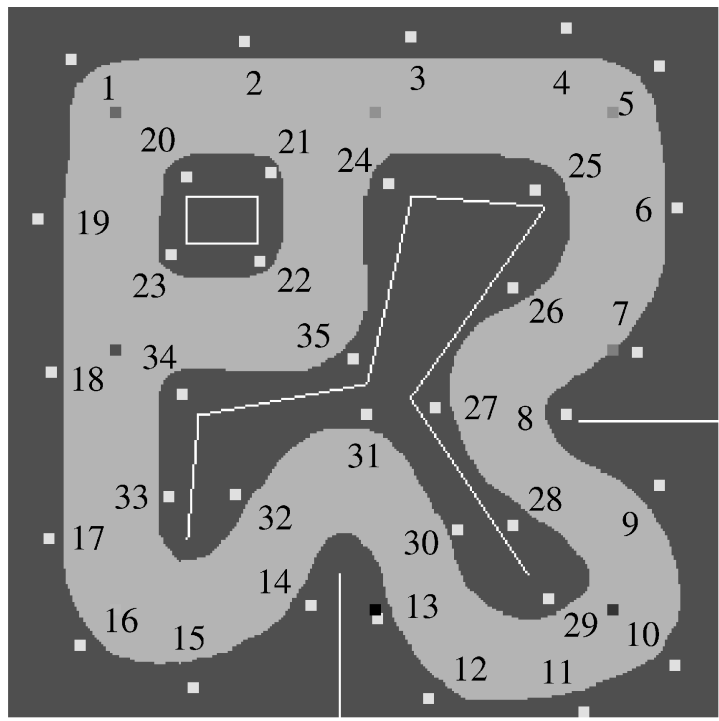

Fig. 17. Complex environment with 35 vision sensors and eight moving objects. The white lines indicate walls.

\subsection{Application to a Complex and Realistic Environment}

Next, we have evaluated the method with a complex and realistic environment. The purpose of this experimentation is to evaluate practicality of the method.

There are 35 vision sensors and eight moving objects in a $30 \mathrm{~m} \times 30 \mathrm{~m}$ space, as shown in Fig. 17 (this is similar to the real environment, as shown in Fig. 3). The objects are identified by their color. In this environment, the number of the components is $\left(\begin{array}{c}35 \\ 3\end{array}\right)=6,545$ and it is estimated that about 540 components will be acquired without constraint propagation.

Fig. 18 shows the average number of acquired components over five runs. Note that the acquisition of the components is accelerated to eight times faster than that in the experiment of Section 5.1, since there are eight moving objects. With 2,000 observations, the method has determined the directions of 513 components without constraint

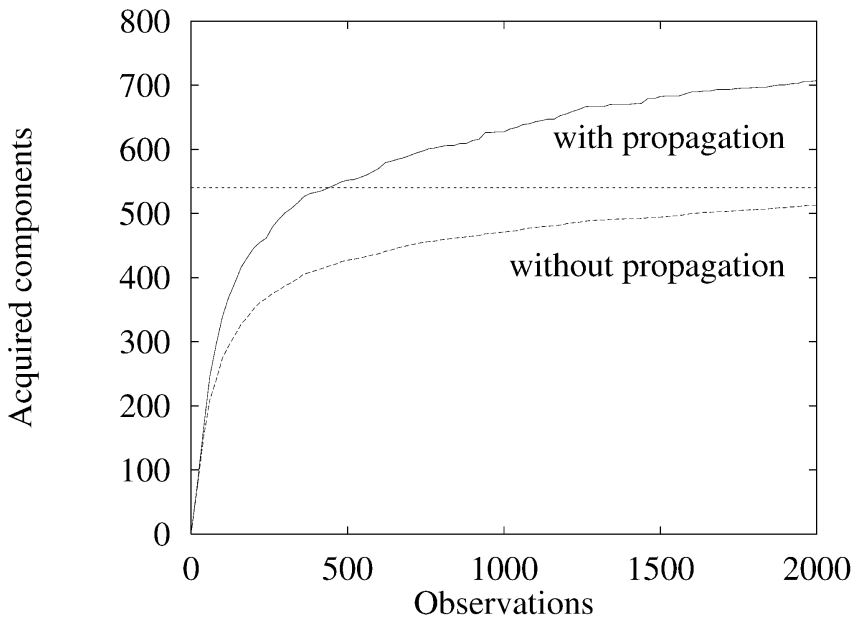

Fig. 18. The number of components acquired with 35 vision sensors in the complex environment. 


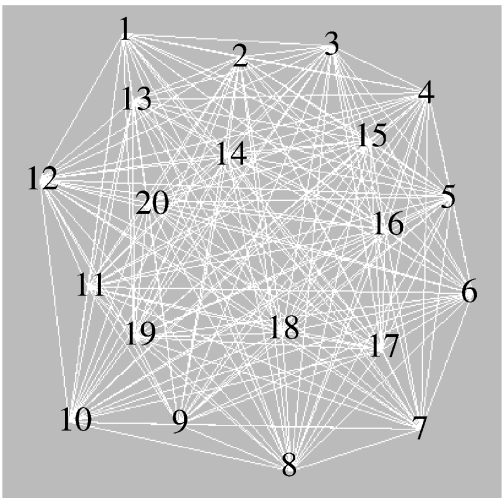

(a)

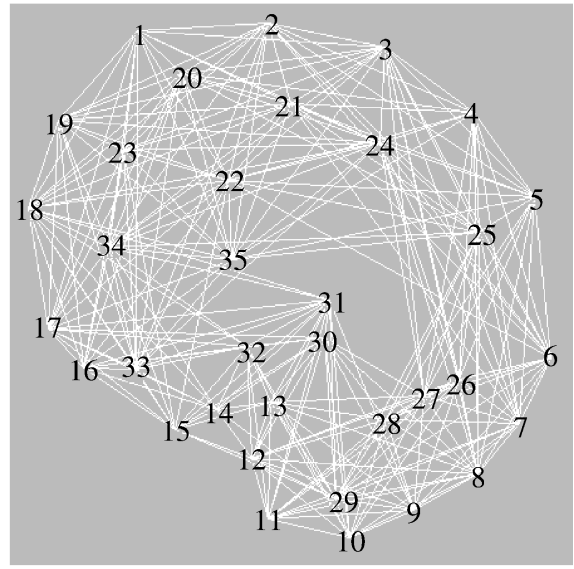

(b)

Fig. 19. Qualitative positions of the vision sensors depicted based on the acquired 3PCs. (a) 20 vision sensors with 5,000 observations. (b) 35 vision sensors with 2,000 observations.

propagation, which is almost equal to the estimated number of 540. With constraint propagation, it has determined the directions of 707 components. In other words, the constraint propagation has acquired about 200 components, which represent the positions of the sensors in distant locations. However, the method could not determine the other components on account of the limitations of observation.

Figs. 19a and 19b show the qualitative positions of the sensors depicted based on the 3PCs acquired in the environments Fig. 15 and 17, respectively. The reason we have used the 3PCs in spite of the components of the qualitative spatial model is that all of the 3PCs cannot be transformed into the components, and they include more constraints than the components. For obtaining Fig. 19, we first located the sensors randomly, then dynamically adjusted the positions by iterating the following steps for arbitrary points, so as to satisfy the constraints of the acquired 3PCs:

1. Compute the force $\mathbf{F}(\propto \exp (d))$ with respect to an arbitrary triangle $A B C$, which moves a point $X$ into a correct region. $d$ is a distance between the current position of $X$ and the correct region (see Fig. 20).

2. Gradually move $X$ based on the resultant of $\mathbf{F}$.

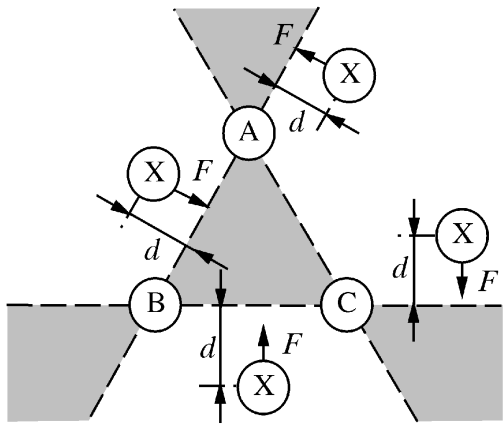

Correct regions for $X$

By comparing Fig. 19a with Fig. 15, and Fig. 19b with Fig. 17, we can find that the acquired positions are topologically correct, that is, the order of connections among the sensors is correct. Although the method could not acquire all of the components, these qualitative maps are sufficient for map-based robot navigation, as shown in Fig. 1a [7], since the qualitative positions of neighboring sensors have been acquired. It can also be used for coarse path planning in the distributed vision system: If we suppose that the sensors (i.e., vision agents) observe the size of the robot, they know which sensor is nearest to the robot, and can determine the order in which they navigate the robot to its destination.

\subsection{Observation Errors}

As described in Section 4.2, observation of motion directions is stable against sensory noise. The proposed method can acquire correct positions of points as long as observed motion directions are correct, however, once a wrong direction is observed, several wrong SCPs and 3PCs may be acquired, which causes inconsistency in the qualitative spatial model. In this section, we consider the observation errors to verify the sensitivity of the acquisition algorithm itself.

First, we have verified the method in a noisy environment, where the configuration of the sensors is the same as Fig. 17, however, approximately 16 percent of motion directions of the objects are mistakenly determined. Fig. 21 shows the average number of acquired components and that of wrong components (i.e., components whose directions ("+" or "-") are mistakenly determined) over five runs. With 2,000 observations, the method has acquired 795 components including 234 wrong components. Note that the number of the acquired components is more than that of the experiments in Section 5.2 since various SCPs have been acquired on account of observation errors.

In order to eliminate wrong 3PCs, we consider the following statistical method. As described in Section 4.2, not all of the seven 3PCs with respect to arbitrary four points should be acquired with observation. If we suppose that the objects randomly move around and various SCPs and 3PCs are equally acquired multiple times by observation, a considerable number of wrong 3PCs can be eliminated by checking the 3PCs acquired relatively less times than others. With this error elimination, the proposed method has 


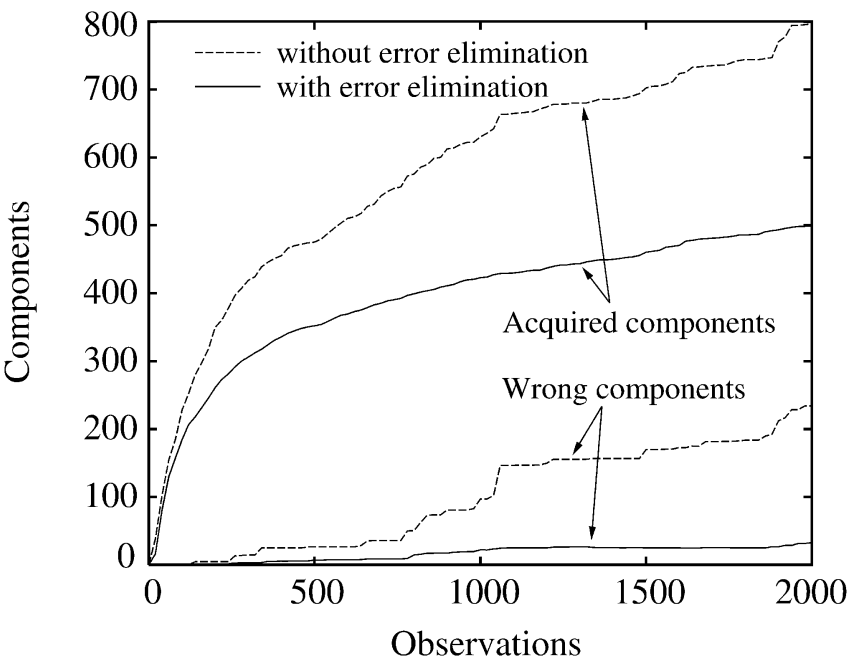

Fig. 21. The number of acquired and wrong components in a noisy environment.

determined 499 components as indicated with "error elimination" in Fig. 21, and reduced the number of wrong components to 32. In other words, the error elimination method could reduce the ratio of wrong components from 29 percent to 6 percent, though it also reduced the total number of acquired components. However, more sophisticated error elimination methods will be necessary since the above method could not eliminate all of the wrong 3PCs.

\subsection{Discussion}

In the experimentation, the proposed method could not acquire the components representing positional relations of sensors 1) in distant locations, 2) behind walls, and 3) in a straight line (e.g., in Fig. 15, sensors 13-15-18, 1-3-4, etc.). It is not easy to acquire all of the components with respect to 1) and 2) on account of the limitations of observation. In addition, if there are concavities in the environment (e.g., several sensors may be surrounded with walls), it will be also difficult to acquire the components. In future work, it should be analyzed how the acquisition process is affected by the structure of the environment. With respect to 3), the qualitative spatial model cannot originally represent such a positional relation, however, it will be estimated from acquired 3PCs that several sensors are located in a straight line.

With respect to observation errors, the statistical method cannot eliminate all of the wrong 3PCs. In addition, it does not check inconsistency among acquired 3PCs, that is, whether the points can be localized so as to satisfy all of the acquired 3PCs. In fact, the positional relations cannot be depicted in the experiment of Section 5.3 on account of inconsistency in the acquired model. Therefore, geometrical approaches, such as triangle constraints [21], should be developed for more effective error elimination. In such methods, backtracking will be necessary in the same way as constraint satisfaction problems [17]. Especially with distributed computing resources, as discussed in Section 3.7, distributed constraint satisfaction problems [22], [23] can be applied to the error elimination.

\section{Conclusion}

This paper has proposed a method for acquiring a qualitative spatial representation from qualitative motion information of moving objects. Key points of this paper are:

- Qualitative positions of landmarks are acquired from motion directions of objects, which are purely qualitative information and obtained with stable observation.

- With constraint propagation, the positions of landmarks in distant locations can be acquired if sensors are partially observable.

We have presented with simulations that the method is valid for acquiring the qualitative positions of multiple vision sensors.

Finally, we discuss the remaining problems. In the simulations, we shave proposed using omnidirectional vision sensors. If normal vision sensors are used instead of them, there will be some "blind spots" where they cannot simultaneously observe an object, which makes it difficult to acquire the qualitative spatial model with the proposed method on account of insufficient observation. However, the method can acquire the qualitative positions as long as the sensors simultaneously observe motion directions of the object somewhere in the environment. In future work, the condition of visual angles of sensors needed to acquire the qualitative spatial model should be clarified.

The correspondence problem of multiple objects should also be addressed. In a real environment, it is difficult to identify many objects, especially when the positions of the sensors are unknown. Therefore, the correspondence errors should also be checked with the elimination method of observation errors.

\section{References}

[1] H.-H. Nagel, "Image Sequences-Ten (octal) Years-from Phenomenology Toward a Theoretical Foundation," Proc. Int'l Conf. Pattern Recognition, pp. 1174-1185, 1986.

[2] C.B. Madsen and C.S. Andersen, "Optimal Landmark Selection for Triangulation of Robot Position," J. Robotics and Autonomous Systems, vol. 23, no. 4, pp. 277-292, 1998.

[3] J.W. Roach and J.K. Aggarwal, "Determining the Movement of Objects from a Sequence of Images," IEEE Trans. Pattern Analysis and Machine Intelligence, vol. 2, no. 6, pp. 554-562, June 1980.

[4] L. Matthies and S.A. Shafer, "Error Modeling in Stereo Navigation," IEEE J. Robotics and Automation, vol. RA-3, no. 3, pp. 239-248, 1987.

[5] N. Ayache and O. Faugeras, "Maintaining Representations of the Environment of a Mobile Robot," IEEE Trans. Robotics and Automation, vol. 5, no. 6, pp. 804-819, 1989.

[6] T.J. Broida, S. Chandrashekhar, and R. Chellappa, "Recursive 3D Motion Estimation from a Monocular Image Sequence," IEEE Trans. Aerospace and Electronic Systems, vol. 26, no. 4, pp. 639-656, 1990.

[7] T.S. Levitt and D.T. Lawton, "Qualitative Navigation for Mobile Robots," Artificial Intelligence, vol. 44, pp. 305-360, 1990.

[8] W.K. Yeap, "Towards a Computational Theory of Cognitive Maps," Artificial Intelligence, vol. 34, pp. 297-360, 1988.

[9] B.J. Kuipers and Y.-T. Byun, "A Robot Exploration and Mapping Strategy Based on a Semantic Hierarchy of Spatial Representations," J. Robotics and Autonomous Systems, vol. 8, pp. 47-63, 1991.

[10] T. Sogo, H. Ishiguro, and T. Ishida, "Acquisition of Qualitative Spatial Representation by Visual Observation," Proc. Int'l Joint Conf. ArtificiaI Intelligence, pp. 1054-1060, 1999. 
[11] H. Ishiguro, "Distributed Vision System: A Perceptual Information Infrastructure for Robot Navigation," Proc. Int'l Joint Conf. ArtificiaI Intelligence, pp. 36-41, 1997.

[12] T. Sogo, H. Ishiguro, and T. Ishida, "Mobile Robot Navigation by Distributed Vision Agents," Approaches to Intelligent Agents, H. Nakashima and C. Zhang, eds., pp. 96-110, Berlin: Springer, 1999.

[13] H. Ishiguro, "Development of Low-Cost Compact Omnidirectional Vision Sensors and Their Applications," Proc. Int'l Conf. Information Systems, Analysis, and Synthesis, pp. 433-439, 1998.

[14] K.D. Forbus, P. Nielsen, and B. Faltings, "Qualitative Spatial Reasoning: TheCLOCK Project," Artificial Intelligence, vol. 51, pp. 417-471, 1991.

[15] C. Freksa, "Using Orientation Information for Qualitative Spatial Reasoning," Theories and Methods of Spatio-Temporal Reasoning in Geographic Space, A.U. Frank, I. Campari, and U. Formentini, eds., pp. 162-178, Berlin: Springer, 1992.

[16] L. Latecki, R. Röhrig, "Orientation and Qualitative Angle for Spatial Reasonning," Proc. Int'l Joint Conf. Artificial Intelligence, pp. 1544-1549, 1993.

[17] A. Isli and A.G. Cohn, "A New Approach to Cyclic Ordering of 2D Orientations Using Ternary Relation Algebras," Artificial Intelligence, vol. 122, pp. 137-187, 2000.

[18] C. Schlieder, "Reasoning about Ordering," Proc. Int'l Conf. Spatial Information Theory, pp. 341-349, 1995.

[19] N. Alon and E. Györi, "The Number of Small Semispaces of a Finite Set of Points in the Plane," J. Combinatorial Theory, Series A, vol. 41, no. 1, pp. 154-157, 1986.

[20] T.K. Dey, "Improved Bounds for Planar $k$-sets and Related Problems," Discrete and Computational Geometry, vol. 19, no. 3, pp. 373-382, 1998

[21] H.-K. Kim, "Qualitative Kinematics of Linkages," Recent Advances in Qualitative Physics, B. Faltings and P. Struss, eds., pp. 137-151, London: MIT Press 1992.

[22] M. Yokoo, E.H. Durfee, T. Ishida, and K. Kuwabara, "The Distributed Constraint Satisfaction Problem: Formalization and Algorithms," IEEE Trans. Knowledge and Data Eng., vol. 10, no. 5, pp. $673-685$, Sept./Oct. 1998

[23] M. Yokoo and T. Ishida, "Search Algorithms for Agents," Multiagent Systems: A Modern Approach to Distributed Artificial Intelligence, G. Weiss, ed., pp. 165-199, MIT Press, 1999.

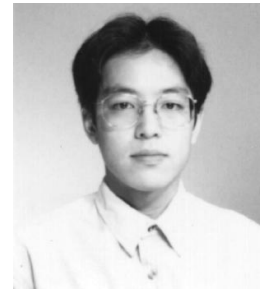

Takushi Sogo received the BEng and MEng degrees from Kyoto University, Kyoto, Japan, in 1997 and 1998, respectively. He is currently a research fellow for the Japan Society for the Promotion of Science and he is working toward his $\mathrm{PhD}$ degree in informatics at the same university. His current research interests include vision system and autonomous mobile robot. $\mathrm{He}$ is a student member of the IEEE.

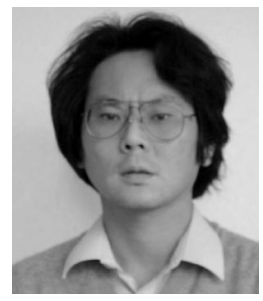

Hiroshi Ishiguro received the BS and MS degrees from Yamanashi University, Japan, in 1986 and 1988, respectively, and the DEng degree from Osaka University, Japan, in 1991. $\mathrm{He}$ is currently an associate professor in the Department of Computer and Communication Sciences at Wakayama University, Japan. He was a visiting researcher in the Electrical and Computer Engineering Department at the University of California, San Diego, from 1998 to 1999. His research interests focus on distributed vision, panoramic vision, active vision, and autonomous mobile robot. He is a member of the IEEE.

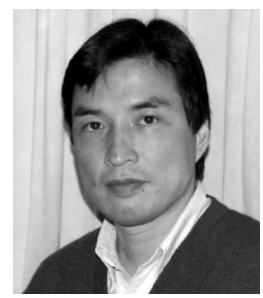

Toru Ishida received the BEng, MEng, and DEng degrees from Kyoto University, Kyoto, Japan, in 1976, 1978, and 1989, respectively. $\mathrm{He}$ is currently a professor in the Department of Social Informatics, Kyoto University, Kyoto, Japan. From 1978 to 1993, he was a research scientist of NTT Laboratories. He was also a visiting research scientist in the Department of Computer Science, Columbia University, an invited professor at Le Laboratoire d'Informatique de Paris 6 (LIP6), Pierre et Marie Curie, and a guest professor at the Institut fuer Informatik, Technische Universitaet Muenchen. He has been working on "parallel, distributed, and multiagent production systems" (Springer, 1994) since 1983. He first proposed parallel rule firing and extended it to distributed rule firing. Organizational self-design was then introduced into distributed production systems for increasing adaptiveness. In 1990, he started working on Real-Time Search for Learning Autonomous Agents (Kluwer Academic, 1997). Again, organizational adaptation becomes a central issue in controlling multiple problem solving agents. He began working on communityware in 1995 and has edited two books: Community Computing: Collaboration over Global Information Networks, (John Wiley and Sons, 1998), and Community Computing and Support Systems, (Springer-Verlag, 1998). $\mathrm{He}$ is currently working on Digital Cities: Experiences, Technologies, and Future Perspectives (Springer, 2000) and initiated Digital City Kyoto with his colleagues. He is a senior member of the IEEE. 